\title{
Intersections
}

Canadian Journal of Music

Revue canadienne de musique

\section{La Pensée de Lévi-Strauss comme objet esthétique}

\section{Jean-Jacques Nattiez}

Volume 30, numéro 1, 2010

URI : https://id.erudit.org/iderudit/1003497ar

DOI : https://doi.org/10.7202/1003497ar

Aller au sommaire du numéro

\section{Éditeur(s)}

Canadian University Music Society / Société de musique des universités canadiennes

ISSN

1911-0146 (imprimé)

1918-512X (numérique)

Découvrir la revue

Citer cet article

Nattiez, J.-J. (2010). La Pensée de Lévi-Strauss comme objet esthétique. Intersections, 30(1), 23-28. https://doi.org/10.7202/1003497ar

\section{Résumé de l'article}

L'auteur rappelle tout d'abord quelques-unes des manifestations publiques de l'engouement pour l'oeuvre de Lévi-Strauss, des Tristes Tropiques à aujourd'hui, y compris le témoignage inattendu d'un garagiste. Adoptant une position résolument critique par rapport à l'entreprise structuraliste dont l'anthropologue s'est fait le porte-drapeau, il essaie d'expliquer le succès de Lévi-Strauss par les propriétés esthétiques de sa pensée. S'inspirant pour cela d'une observation de Jean Molino, il rappelle que les catégories de l'entièreté, de l'harmonie et de l'éclat se rencontrent aussi bien chez les philosophes platoniciens et néo-platoniciens que chez un esthéticien américain contemporain. Cette permanence dans le temps peut faire penser que l'on tient là quelques-unes des familles universelles qui regroupent les critères du Beau. Il montre alors qu'elles peuvent rendre compte aussi bien du mode de pensée lévi-straussien que de l'oeuvre de Richard Wagner, ce qui permet d'expliquer, du même coup, la fascination de Lévi-Strauss pour ce compositeur.
All Rights Reserved (C) Canadian University Music Society / Société de musique des universités canadiennes, 2011
Ce document est protégé par la loi sur le droit d'auteur. L'utilisation des services d'Érudit (y compris la reproduction) est assujettie à sa politique d'utilisation que vous pouvez consulter en ligne.

https://apropos.erudit.org/fr/usagers/politique-dutilisation/ 


\title{
LA PENSÉE DE LÉVI-STRAUSS COMME OBJET ESTHÉTIQUE
}

\author{
Jean-Jacques Nattiez
}

L'histoire se passe dans un garage. Je viens y porter mon automobile pour la révision hivernale. Le préposé, en bleu de chauffe et les doigts un peu sales, me demande mon nom. Il cherche mon dossier dans son ordinateur, il me regarde et me dit : "C'est vous qui avez écrit un livre sur Lévi-Strauss et la musique?» Un point d'interrogation s'inscrit au milieu de ma figure. "Oui. Comment savez-vous cela?» "Eh bien, précise-t-il, j'ai lu il y a quelques mois l'édition des Euvres de Lévi-Strauss en Pléiade. J’ai voulu en savoir plus sur cet anthropologue. J'ai tapé «Lévi-Strauss» sur Google et je suis tombé sur votre nom ...» Je le questionne sur sa lecture. "J'ai beaucoup aimé Tristes tropiques. Mais honnêtement, ses analyses de mythes, je trouve cela un peu difficile, mais c'est toujours très beau... » Le tout au milieu des taches de graisse, des bidons d'essence et des piles de pneus usagés.

Cette histoire est vraie. Je la rapporte pour trois raisons. D’abord, je la trouve émouvante, ce qui se passe de commentaires. Ensuite, elle est positivement dérangeante : n'avons-nous pas trop tendance à considérer que seuls les gens d'une certaine formation intellectuelle ont spontanément accès à ce que l'on appelle parfois la «haute-culture»? Ensuite, parce qu'il convient de se demander ce qui peut bien avoir provoqué l'intérêt a priori d'un travailleur manuel pour l'œuvre et la pensée de Lévi-Strauss. Probablement, quelques-unes des raisons que je vais réunir ici.

Lorsque, en mai 2008, les éditions Gallimard ont publié une vaste sélection des écrits de Lévi-Strauss dans la Bibliothèque de la Pléiade, elles ont réussi un " coup» magnifique, comme on dit dans le métier. Elles ont devancé l'incroyable déferlement médiatique provoqué, en France, par les célébrations du centenaire de sa naissance le 28 novembre de la même année. Quinze mille exemplaires immédiatement vendus, et cette parution coiffe au poteau le quarantième anniversaire des événements du 13 Mai 1968 : c’est le Pléiade de Lévi-Strauss qui, cette semaine-là, fait l'objet de la couverture du Nouvel Observateur! À dire vrai, le phénomène n'est pas nouveau. La parution de Tristes tropiques, en 1955, avait déclenché en 1955 un engouement certain (100 ooo lecteurs), provoquant une prise de conscience anticolonialiste, la découverte de cultures autres, en même temps que ce livre révélait à un large public l'existence et l'importance des sciences humaines. Le tout dans le style d'un grand écrivain : bien qu'il ne s'agisse pas d'un roman, il fut un moment sérieusement considéré pour le Prix Goncourt. Par la suite, tout nouveau livre de Lévi-Strauss était attendu avec autant d'impatience que le dernier Tintin, et a souvent entraîné la parution de 
numéros spéciaux de revues que fréquentent les intellectuels français comme L'Arc, Critique, Esprit ou Le magazine littéraire. Moi-même, aux beaux temps du structuralisme triomphant, et à l'époque où la sémiologie musicale cherchait à se définir à partir et au-delà du structuralisme, j’ai sacrifié à ce rituel en préparant en 1973 le numéro 12 de la revue Musique en jeu consacré aux rapports de Lévi-Strauss et de la musique.

Plusieurs raisons peuvent expliquer le rayonnement du "phénomène Lévi-Strauss», y compris jusque dans l'officine de mon garagiste. Tout d'abord, l'ampleur de l'œuvre : vingt livres, pour s'en tenir aux éditions françaises, sans parler de la myriade d'articles qui les prépare ou en prolonge le contenu, les innombrables entretiens journalistiques qui les accompagnent et les articles de presse qui amplifient la rumeur que tout cela déclenche. Mais le nombre, en lui-même, n'explique rien. L'œuvre de Lévi-Strauss développe à la fois une philosophie et une méthodologie qui ambitionne de donner du fonctionnement humain une explication holiste. De la même façon qu'il y a un Homme marxien et un Homme freudien, il y a un Homme structural. Une des raisons pour laquelle le centenaire de Lévi-Strauss a déclenché tant d'événements, y compris la journée d'études de la Faculté de musique de l'Université de Montréal dont ce numéro se fait l'écho, c'est parce que l'on pensait que sa pensée ouvrait la porte à une meilleure compréhension globale de l'humanité et des cultures. Et cela, pas seulement en élaborant une philosophie originale de notre nature et de notre destinée, mais en proposant une méthode aux ambitions et aux objectifs scientifiques qui puisse être relayée par de nouvelles générations de chercheurs : anthropologues, sociologues, théoriciens de la littérature, esthéticiens, et même musicologues. Ajoutons à cela que, très vite, Lévi-Strauss prit position par rapport à des questions de société et de philosophie dans des domaines aussi divers que les causes du racisme, la validité du marxisme (ou de l'existentialisme), les préoccupations écologiques, l'avenir des civilisations et l'esthétique, notamment en se prononçant sur le statut et la valeur de l'art abstrait et de la musique contemporaine. Dès lors, toute prise de position de Lévi-Strauss, dans quelque domaine que ce soit, bénéficiait de l'aura d'un remarquable écrivain, du prestige d'un grand penseur et du crédit attribué a priori à un chercheur scientifique. De ce fait, elle pouvait devenir l'objet d'intenses discussions tous azimuts, qu'il s'agisse des réactions houleuses et multiples aux prises de position de Lévi-Strauss contre la musique concrète et la musique sérielle (Lévi-Strauss 1964, p. 26-34), ou de l'interprétation que l'on peut donner du parrainage wagnérien dont Lévi-Strauss s'est réclamé (ibid., p. 23). Il en a été, à nouveau, abondamment question dans les pages qui précèdent. Il prend l'envie d'appliquer à Lévi-Strauss le mot de Thomas Mann au sujet de Wagner : «Wagner und kein Ende.» Transposons : avec Lévi-Strauss, il n’y a jamais de fin ...

Maintenant que les déferlements médiatiques du centenaire de Lévi-Strauss se sont estompés et qu'ils ont connu un dernier soubresaut avec son décès le 30 octobre 2009, il me semble nécessaire, comme j'ai tenté de le faire dans mon Lévi-Strauss musicien (Nattiez 2008), d'adopter vis-à-vis de son œuvre et de sa pensée une attitude beaucoup plus critique que trop de chercheurs et 
d'intellectuels ne l'ont fait, sans doute impressionnés par le charisme holiste de sa pensée. Il faut tout d'abord affirmer avec force que bien des prises de position qui ont fait débat, notamment celles portant sur l'art et sur la musique, n'ont pas un lien direct avec la méthodologie du structuralisme telle que l'anthropologue l'a élaborée et développée. Je parle bien de son structuralisme à lui car, comme Raymond Boudon y a insisté, il n'y a pas un mais des structuralismes, selon que ce qui est considéré a priori comme objet-système est défini ou indéfini, et selon que la théorie utilisée est vérifiable, indirectement vérifiable ou invérifiable (Boudon 1968, p. 103). Plus Lévi-Strauss a avancé en âge, plus il a reconnu la subjectivité dont émanent ses prises de position.

Mais ce n'est pas tout. Je voudrais repartir ici du témoignage de mon garagiste. Pourquoi la pensée de Lévi-Strauss est-elle attirante et séduisante? C’est lui qui déclare sans ambages que, même quand il ne comprend pas bien les analyses de mythes, il les trouve belles. Je ne lui reprocherai certainement pas de ne pas avoir interrogé la méthodologie de l'analyse structurale des mythes, alors que les chercheurs qui se sont sérieusement penchés sur le problème se comptent sur les doigts d'une main. Et soyons méchants : qui, des journalistes aux dissertations prolifiques du Nouvel Observateur, du Monde ou de L'Express, en 2008 et en 2009; qui, de leurs lecteurs et de leurs lectrices, serait vraiment capable de donner une définition simple et synthétique du structuralisme et de ses principes méthodologiques. La question qu'il aurait fallu se poser dès la parution de l'article historique consacré à «La structure des mythes» (Lévi-Strauss 1955), puis à chacun des volumes de l'immense «tétralogie» des Mythologiques, aurait été d'examiner, dans la perspective définie par l'archéologue Jean-Claude Gardin - ce qu'il appelle «l'analyse logiciste» (Gardin 1974 et 1979, passim) - quelles sont les procédures selon lesquelles le mythographe définit les unités signifiantes des mythes et sur la base de quelles opérations de l'esprit il les regroupe et les interprète. Tant qu'on ne répond pas à ces questions méthodologiques et épistémologiques de base, on ne fait, à mon sens, que contempler et admirer l'élégance d'une construction intellectuelle toujours fascinante, parfois audacieuse, voire paradoxale, mais trop souvent hasardeuse.

Or, il y a une raison de fond pour laquelle l'application de l'investigation structuraliste à tel ou tel objet, qu'il s'agisse d'un leitmotiv particulier de la Tétralogie de Wagner ou de l'organisation du Boléro de Ravel, capte notre intérêt. Fondamentalement, Lévi-Strauss cherche à établir entre les unités prises en considération des relations d'équivalence, de symétrie et d'inversion. Or, si on ne se donne pas de critères un tant soit peu rigoureux pour définir en quoi consistent ces trois familles de relations, la confiance que l'on peut attribuer au modèle proposé pour rendre compte de l'objet étudié s'avère rapidement limitée. À moins de considérer, simplement, que la construction élaborée par Lévi-Strauss est belle. Or elle l'est.

Dans un important texte consacré aux fondements du jugement esthétique, Jean Molino a comparé les «conditions objectives du Beau» telles qu'ont été proposées par les platoniciens et les néo-platoniciens, avec celles d'un esthéticien américain contemporain, Monroe Beardsley. Il résume ainsi les premières : 
1. l'entièreté (oui, le mot existe!) ou intégrité, integritas sive perfectio des Scolastiques; l'objet beau doit être déterminé par une forme correspondant à son type pleinement réalisé. C'est pourquoi le critère de l'entièreté pour un être est de ne pas manquer de tout ce qu'il devrait avoir : un objet beau, c'est un objet auquel il ne manque rien;

2. l'harmonie, harmonia, définie par Plotin comme «l'accord dans la proportion des parties entre elles et avec le tout» (Ennéades, I, 6, 1);

3. l'éclat, claritas, qui correspond au plaisir procuré par les qualités sensibles, à ce qui, dans l'objet, 'saisit et retient le regard'. (Gilson 1963, p. 49) (Molino 2009, p. 356).

Il examine ensuite comment Beardsley (1958, in 1981, p. 466) définit les «canons généraux» de la Beauté par rapport auxquels on peut classer

les diverses raisons objectives de la valeur d'une œuvre d'art, quel que soit le genre auquel elle appartient, peinture, musique, poésie :

1) le canon d'unité;

2) le canon de complexité;

3) le canon d'intensité, c'est-à-dire l'intensité de "qualités humaines régionales» - l'œuvre est pleine de vitalité, forte, tendre, ironique, etc. (Molino 2009, ibid.)

J'ai montré ailleurs que l'on pouvait retrouver ces trois familles de critères esthétiques dans des musiques aussi différentes que celles qui accompagnent une danse ougandaise d'initiation au mariage que Répons de Pierre Boulez (Nattiez 1999, p. 231-233).

Je voudrais suggérer ici que, si l'œuvre empirique de Lévi-Strauss - et je pense essentiellement à ses analyses de mythes qui l'ont occupé toute sa vie, de 1955 à 1991 - a séduit sans que l'on n'éprouve le besoin de vérifier pas à pas comment il avait procédé pour construire le modèle qui en était proposé, c'est parce qu'il répond, lui aussi, aux classes de canons esthétiques généraux résumés ci-dessus. (Il en existe probablement d'autres.)

L'objectif même du structuralisme est de faire apparaître l'unité et l'entièreté de l'objet considéré, et ce, en recourant, ad nauseam, aux relations d'équivalence, de symétrie et d'inversion. Grâce à elles, tout se tient, de manière homogène, et elles ne laissent rien de côté, puisque, lorsqu'une discordance apparaît, il suffit de l'expliquer par l'inversion.... Le structuralisme parvient ainsi à considérer les faits qu'il étudie comme des totalités. En lisant les analyses de mythes par Lévi-Strauss, c'est cette même fascination pour l'unité, l'équilibre et l'organisation élégante d'une œuvre d'art que nous éprouvons.

Le structuralisme lévi-straussien n'est pas désarmé devant la complexité des phénomènes. Je dirais même qu'elle le stimule, puisque, face, par exemple, au millier de mythes sud-américains pris en charge dans les quatre volumes des Mythologiques, l'objectif est de ramener leur complexité de surface à des catégories sensibles qui, de proche en proche et par glissements sémantiques successifs, les rattachent à ce que Lévi-Strauss considère comme des structures universelles de l'esprit humain : le cru et le cuit, le rôti et le bouilli, le chaud et le froid, le masculin et le féminin, etc.

Tout cela ne manque pas d'éclat ou d'intensité, car, lorsque, dans les dernières pages de L'Homme $n \mathcal{u}$, l'auteur ramène toutes les oppositions binaires qu'il 
a patiemment exhibées au cours des deux mille pages précédentes, à l'opposition de l'être ou du non-être, le frisson métaphysique qui s'empare de nous en nous faisant toucher du doigt, comme par magie, l'alpha et l'oméga de la condition et de la pensée humaines, provoque chez le lecteur et la lectrice une forme d'orgasme intellectuel qui n'est pas sans analogie avec celui, parfois plus concret, auquel peut nous mener la résolution tonale des tensions provoquées par une cascade de chromatismes dans un opéra de Wagner comme Tristan et Isolde.

Et ce n'est pas sans raison que j'introduis ici une comparaison avec Wagner. Car, pour rendre compte de l'impression de beauté et de sublime que certaines de ses œuvres, ou certains passages de ses œuvres déclenchent en nous, ne peut-on pas, aussi, faire appel à ces catégories d'unité, de complexité et d'intensité? Le système des leitmotive est en effet d'une cohérence admirable : en se fondant sur l'analyse des leitmotive par Deryck Cook, il est possible de montrer que tous les motifs diatoniques de L'Anneau du Nibelung peuvent être dérivés les uns des autres, par transformations successives, à partir de l'accord parfait initial de L'Or du Rhin. La complexité? Quoi de plus complexe en effet qu'une œuvre de Wagner dans laquelle, pour s'en tenir au seul aspect musical, on ne cesse de découvrir de nouvelles relations entre chacun des motifs nombreux qui la parcourent? Quant à l'intensité, due à une orchestration dont la puissance paroxystique n'avait jamais été atteinte avant lui, c'est bien elle qui, à la fois, a créé l'envoûtement passionné de cohortes d'admirateurs inconditionnels dans tous les pays et à chaque génération, mais aussi le rejet de ceux et celles qui restent réfractaires à cette force dévastatrice. Les deux attitudes peuvent, du reste, se retrouver chez une même personne comme Nietzsche, mais à des moments différents de sa vie.

Et c'est sans doute dans cette fascination commune, chez Wagner et chez Lévi-Strauss, pour l'unité, la complexité et l'intensité, que, pour ma part, je verrais la raison fondamentale de l'admiration de Lévi-Strauss pour Wagner. C'est aussi celle que lecteurs et lectrices peuvent ressentir à la lecture de ses ouvrages, même les plus ésotériques lorsqu'il s'agit d'analyses de mythes. Et l'auteur de ces lignes ne s'en cache pas: si, malgré mes nombreuses réserves critiques, pour ne pas dire plus, à l'égard de la méthodologie de Lévi-Strauss, j’ai consacré un certain nombre d'heures à étudier sa pensée, c'est probablement parce que, au cours de l'exercice, j'ai éprouvé un authentique plaisir esthétique, celui qui la fait qualifier de belle.

\section{RÉFÉRENCES}

Beardsley, Monroe C. 1958. Aesthetics. New York : Harcourt Brace.

Boudon, Raymond. 1968. À quoi sert la notion de "structure»? Essai sur la signification de la notion de structure dans les sciences humaines. Paris : Gallimard.

Gardin, Jean-Claude. 1974. Les analyses de discours. Neuchâtel : Delachaux et Niestlé.

-1979. Une archéologie théorique. Paris: Hachette.

Gilson, Étienne. 1963. Introduction aux arts du beau. Paris : Vrin. 
Lévi-Strauss, Claude. 1955. "The Structural Study of Myth», in «Myth, a Symposium ", Journal of American Folklore, 78, $\mathrm{n}^{0} 270$ (oct.-déc.) : 248-244. Version française révisée : «La structure des mythes", Anthropologie structurale. Paris : Plon, 428-444.

1964. Mythologiques, Le cru et le cuit. Paris : Plon.

Molino, Jean. 2009. Le singe musicien. Essais de sémiologie et d'anthropologie de la musique. Arles: Actes Sud / Ina.

Nattiez, Jean-Jacques. 1999. La musique, la recherche et la vie. Un dialogue et quelques dérives. Montréal : Leméac.

-2008. Lévi-Strauss musicien. Essai sur la tentation homologique. Arles : Actes Sud.

\section{RÉSUMÉ}

Lauteur rappelle tout d'abord quelques-unes des manifestations publiques de l'engouement pour l'œuvre de Lévi-Strauss, des Tristes Tropiques à aujourd'hui, y compris le témoignage inattendu d'un garagiste. Adoptant une position résolument critique par rapport à l'entreprise structuraliste dont l'anthropologue s'est fait le porte-drapeau, il essaie d'expliquer le succès de Lévi-Strauss par les propriétés esthétiques de sa pensée. S'inspirant pour cela d'une observation de Jean Molino, il rappelle que les catégories de l'entièreté, de l'harmonie et de l'éclat se rencontrent aussi bien chez les philosophes platoniciens et néo-platoniciens que chez un esthéticien américain contemporain. Cette permanence dans le temps peut faire penser que l'on tient là quelques-unes des familles universelles qui regroupent les critères du Beau. Il montre alors qu'elles peuvent rendre compte aussi bien du mode de pensée lévi-straussien que de l'œuvre de Richard Wagner, ce qui permet d'expliquer, du même coup, la fascination de LéviStrauss pour ce compositeur.

\section{ABSTRACT}

The author first recalls some of the public expressions of enthusiasm for the work of Lévi-Strauss, from Tristes Tropiques to today, including the unexpected opinions of a mechanic. Taking a strongly critical position toward the structuralist project for which the anthropologist has become the standard bearer, the author tries to explain the success of Lévi-Strauss through the aesthetic properties of his thinking. Drawing on an observation by John Molino to this end, he recalls that the categories of wholeness, harmony and radiance are found as equally among Platonists and Neoplatonists as among a contemporary American aesthetician. This permanence in time may suggest that there are some universal categories that incorporate the requisites of the beautiful. The author then shows that these can explain both levi-straussian thought and the works of Richard Wagner, which helps explain, at the same time, the fascination of Lévi-Strauss for this composer. 